\title{
Correlative reference model and molecular dynamics simulation of dislocation emission process
}

\author{
Qiheng Tang *, Tzuchiang Wang \\ LNM, Institute of Mechanics, Chinese Academy of Sciences, 15 Zhong Guan Cun Road, Beijing, 100 080, People's Republic of China
}

Received 9 February 1998; accepted 6 June 1998

\begin{abstract}
A correlative reference model for computer molecular dynamics simulations is proposed. Based on this model, a flexible displacement boundary scheme is introduced and the dislocations emitted from a crack tip can continuously pass through the border of the inner discrete atomic region and pile up at the outer continuum region. The effect of the emitted dislocations within the plastic zone on the inner atomistic region can be clearly demonstrated. The simulations for a molybdinum crystal show that a full dislocation in a bec crystal is dissociated into three partial dislocations and interaction between the crack and the emitted dislocations results in gradual decrease of the local stress intensity factor. (c) 1998 Elsevier Science B.V. All rights reserved.
\end{abstract}

\section{Introduction}

Rice and Thomson [1] proposed a criterion for dislocation emission from a stressed crack tip which is quite simple and can be applied to intrinsically brittle materials or intrinsically ductile ones. Sinclair and Finnis [2] extended the Rice and Thomson analysis to address the problem of whether the presence of one or more emitted dislocations affects the competition between further emission and cleavage. Lin and Hirth [3] studied the changes of stress with dislocation nucleation from a crack. Rice [4] used the Peierls concept to reanalyze the dislocation nucleation from a crack tip and proposed a new solid state parameter $\gamma_{u s}$ the unstable stacking energy, to evaluate the critical external loading which

\footnotetext{
${ }^{*}$ Corresponding author. Fax: +86 0106256 1284; e-mail: tcwang@cc5.imech.ac.cn
}

corresponds to dislocation nucleation. Wang [5] extended Rice's analysis with a set of new governing equations which can be employed to analyze the dislocation emission.

The above analyses were unsatisfactory for near crack tip fields because of their use of continuum elasticity for the crack tip stress field. Near the crack tip region, the atomic lattice effect becomes important, and the atomic force law needs to be considered. The experimental results by Choi et al. [6] showed that the core size of dislocation is far beyond that predicated by linear elasticity theory. It is essential to analyze the dislocation nucleation and emission from a crack tip by atomic simulation utilizing an appropriate interatomic force law and the correct crystallographic geometry of simulated specimen.

Bullough [7] and Cotterill [8] made what probably were the first molecular dynamics (MD) calculations for such systems. Born-Mayer and Morse potentials were used to study the lattice 
structure of the dislocation center. Using the embedded atom method (EAM), Baskes et al. [9] investigated dislocation mobility in nickle. Applying 3-dimensional MD method, for the crystal structure of $\mathrm{Cu}$, Zhang et al. [10,11] simulated dislocation emission and dislocation mechanical behavior near a crack tip. A fixed displacement boundary condition was employed in their simulations.

As pointed out by Cheung et al. [12], the proper choice of the boundary conditions on the border of the inner discrete atomic region is of crucial important for the accuracy of the final results. Generally speaking, there are three types of boundary schemes in MD simulations: the fixed displacement boundary scheme, the flexible displacement boundary scheme and the force (stress) boundary scheme. The fixed displacement boundary condition has been used by many authors $[13,14]$. The positions of the atoms on the border are given according to linear elastic theory and subsequently held fixed as the outer materials are removed during the simulation. Incompatibilities at the border between the inner discrete atomic region and the outer continuum region will arise unless a flexible displacement boundary scheme is employed. Various versions of the flexible displacement boundary scheme have been proposed $[15,16]$. In this case, the atoms forming the border of inner system are given appropriate positions characteristic of the outer region. As the discrete interior region, still attached to the outer continuum, relaxes during the simulation, forces are generated on the atoms in the border region. These forces are then relieved in an iterative fashion through the use of a Green's function derived from linear elasticity theory. The third type of boundary scheme is a set of forces (or stresses) that are prescribed along the border of the discrete atom region. These forces are obtained from an appropriate linear elastic solution. The outer region is then removed while the forces are maintained on the border atoms throughout the simulation.

A particularly serious deficiency resulting from both the fixed boundary and the flexible boundary with use of the Green's function is that they do not allow the dislocation to pass through the border. The force specified boundary condition offers no resistance to the penetration of a dislocation through the border into the outer region, but it gives dynamically too complex results to describe the essence of the fracture process [17]. The typical size of a crystal modeled on the atomic level in a computer simulation study is restricted to the order of ten or hundred thousand atoms by the cost of the computation. Often this is too small for the atomic level simulation to reveal the complex mechanical properties of the materials.

A finite element model combined with atomistic modeling was proposed by Mullins and Dokainish [18], with shape function used to perform the transition of stress (or force) and displacement between the continuum and the atomistic regions. In their works, the effect of different crystal orientations on crack propagation has been studied [18]. The behavior of a crack at the atomic scale is quite complex under mixed mode loading [19]. The transition region of the lattice and the continuum was divided into two zones by Kohlhoff et al. [20], with each zone providing the displacement boundary condition for the other. Elastic constants limited to third-order in the continuum region were required to be equal to those defined by the interatomic potential in the atomistic region. Based on this model, Gumbsch [21] found that brittle cracks under general mixed model loading situations follow an energy criterion rather than an opening-stress criterion. Tan and Yang [22] realized transmissions of some mechanical parameters, such as displacements, stresses and mass in the overlapping belt of atomistic core and the continuum, but a simple lattice was adopted in their simulation. In the field of atomistic core and continuum studies, a common difficulty in all hybrid models [18-22] is the proper treatment of the transition between the lattice and continuum. The problem arises form the different nature of the internal forces which act on the two regions.

A correlative reference model is proposed in this paper. Based on this model, a new flexible displacement boundary scheme for atomic level computer simulations is developed which provides several advantage over the previous boundary schemes.

1. It offers no resistance for the emitted dislocation to penetrate through the border. 
2. It eliminates the instability problem encountered with the specified force boundary method.

3. By the new flexible boundary scheme, the effects of the emitted dislocations pileup in the outer continuum region on the atomic motion in the inner region can be clearly demonstrated.

\section{Mechanical model}

The correlative reference model is schematically shown in Fig. 1. Fig. 1(a) shows a simulated sample of the MD approach, with a single crack. Region 1 comprises the inner discrete atoms, and region 2 is the border of the inner simulated atoms. $S_{\mathrm{R}}$ represents an emitted dislocation. Fig. 1(b) shows an infinite continuum with a single edge crack, its elastic constants, such as $E, G, v$ are the same as those of the simulated Mo crystal. The geometrical configuration and the size outlined by the dotted line in Fig. 1(b) is also the same as that of Fig. 1(a). This infinite elastic continuum is designated as a reference model and is subject to remote loading. There is a correlation between the realistic simulated sample and the reference model. Two requirements must be satisfied in the simulation:

1. The displacements of the atoms in region 2 in Fig. 1(a) are imposed by displacements of the corresponding region in the reference model.

2. Supposing that there are $N_{\mathrm{T}}$ dislocations emitted from the crack tip, here $N_{\mathrm{T}}=N_{1}+N_{3}$. The positions of the $N_{1}$ emitted dislocations in region 1 of Fig. 1(b) are defined by the discrete atomic configuration in Fig. 1(a). Other $N_{3}$ dislocation positions in the continuum region 3 are determined by the dislocation pileup theory of mescromechanics.

\subsection{Simulation procedure}

Flexible displacement scheme: Firstly consider the problem of the reference model, the pure $K_{\mathrm{II}}$ stress field is prescribed at infinity and the stress intensity factor $K_{\mathrm{II}}$ is chosen as a load parameter. Suppose the slip plane is coincident with the crack plane and the slip direction is along $x$ axis in the present study. Hence all emitted dislocations lie on the $x$ axis. Based on linear elastic theory, the displacement field of the infinite continuum with a single edge crack and $N_{\mathrm{T}}$ discrete dislocations is given by the following equation

$u_{l}=u_{l}^{A}+\sum_{i=1}^{N_{\mathrm{T}}} u_{l}^{D}\left(x, y ; x_{i}\right)$

where $l$ represents coordinate components in the $x$ and $y$ directions, $x_{i}$ is the position of the $i$ th discrete dislocation, both $u_{l}^{A}$ and $u_{l}^{D}$ are the displacement fields corresponding to the applied remote load and to the dislocation, respectively. The displacement $u_{l}$ depends not only on the applied remote load but also on the emitted dislocations.

Before any dislocation emission, the displacement field in the reference model is just the pure $K_{\text {II }}$ field, and the displacements imposed on the atoms in the border of the discrete atomic system are also the pure $K_{\text {II }}$ field. Hence the present boundary condition is actually the same as the fixed displacement boundary condition until the first full dislocation is nucleated.

When a dislocation is nucleated in the crack tip region which is detected from the MD simulation on the discrete atomic system (see Fig. 1(a)), in the reference model, we will pause to load temporarily and to keep the remote load $K_{\text {II }}$ unchanged. The displacements of the atoms on the border in Fig. 1(a) which are given by the displacements in region 2 of the reference model are held fixed temporarily, and then the discrete atomic system is relaxed.

Because of the unstable characteristics of the emitted dislocation near the crack tip, it is impossible for the nucleated dislocation to stay at a crack tip, and thus the dislocation $S_{\mathrm{R}}$ moves away from the crack tip. Although the external load at infinity is held fixed, the displacement fields will vary with the dislocation emission or movement which offers displacement increment to the deformation of the reference model, and the displacement field imposed on the border of the discrete atomic system in Fig. 1(a) is also changed during the relaxation process of the MD simulation. Therefore the present boundary condition, prescribed on the border of MD simulated sample is 

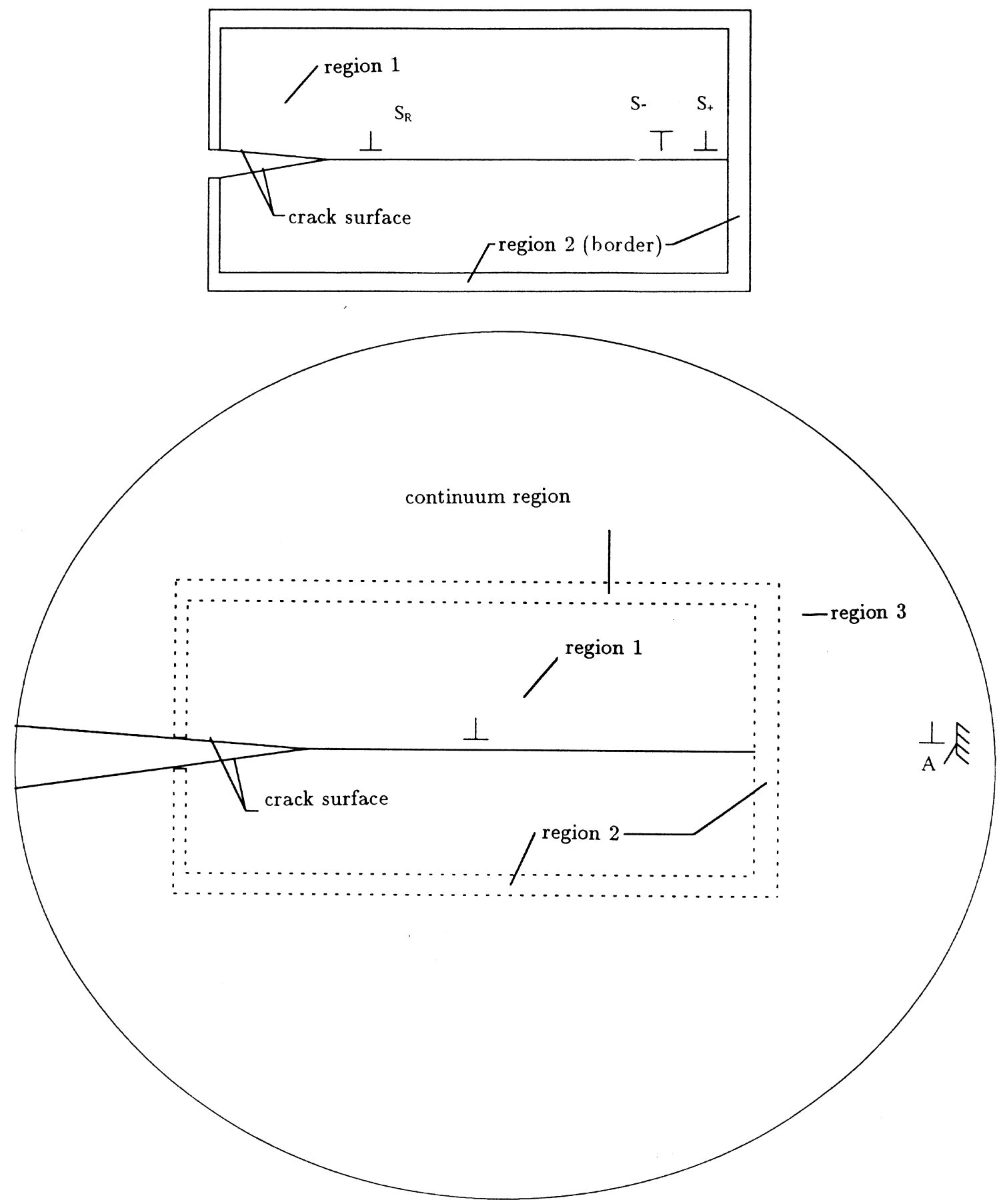

Fig. 1. Schematic diagram for the correlative reference model (a) Schematic diagram for the simulated sample (b) Schematic diagram for the infinite continuum with a single edge crack. 
essentially a flexible displacement boundary condition. The emitted dislocation will pass through the border. If there are still some dislocations in the discrete atomic region 1 , relaxation will be carried on continuously; otherwise, we will load at infinity again with the same loading rate as before in the reference model.

Dislocation penetrating border: Numerous experimental observations have shown that a dislocation will be emitted from the stressed crack tip when the applied stress intensity factor is greater than $K_{\mathrm{II}_{e}}$, and the dislocation can be emitted continuously when the applied load increases slightly. Experiments also show that the emitted dislocations move along the slip plane, and are piled up in front of an obstacle at a distance of several micrometers from the crack tip, where there might be a grain boundary or other impurities or imperfections.

In our simulation, we suppose that the first emitted dislocation will pile up at point $A$, which is at the distance of $5000 a_{0}$ from the crack tip, far beyond the relatively small size of the present sample. A satisfactory result can not be obtained unless the emitted dislocations can penetrate through the border into the continuum region smoothly. Assume that both positive and negative dislocations, marked by $S_{+}$and $S_{-}$, respectively, are near the border. As the emitted dislocation $S_{\mathrm{R}}$ slips to the border and interacts with dislocation $S_{-}$, dislocations $S_{\mathrm{R}}$ and $S_{-}$will be annihilated. The dislocation $S_{+}$is left only near the border and its velocity is the same as that of the emitted dislocation $S_{\mathrm{R}}$, because it is replaced by dislocation $S_{+}$ in region 1. Moreover as $S_{+}$penetrates through the border, it's velocity should be evaluated adequately, based on the velocity of the emitted dislocation. Because the border is no longer an obstacle to affect dislocation motion, the width of the border is very short and the velocity of the emitted dislocation $S_{\mathrm{R}}$ is nearly constant beyond a distance of about $5 a_{0}$ from the crack tip. Thus in our simulation, the velocity of dislocation $S_{+}$is the same as the velocity of the emitted dislocation. When the dislocation $S_{+}$finishes its passage of the border into region 3, a step has been formed on the right border. After that the effect of the movement of the emitted dislocation $S_{+}$on the positions of the atoms on the border becomes much smaller. A common problem in the field of atomic core and continuum studies is the formation of inside step $B_{\mathrm{I}}$ on border 2 which is thrust into atomic core region 1 see Fig. 2(c). The problem is independent of the choice of what type of boundary scheme is employed either the force boundary or the flexible displacement boundary scheme. As soon as the dislocation penetrated through the border 2 , an inside step $B_{\mathrm{I}}$ will be formed, contributed by the relative slip of upper atomic block with respect to the lower atomic block along the slip plane. A changeable border is adopted, atoms in the step region $B_{\mathrm{I}}$ should be changed as atoms of the inner discrete system after the dislocation $S_{+}$penetrates through the border. At the same time, the thermal energy of the discrete atomic system is modified to maintain an isothermal relaxation process.

Positions of dislocations of region 3: The positions of $N_{3}$ emitted dislocations in region 3 of Fig. 1(b) can be determined by the following equations:

$x_{1}=x_{O b}, \quad \tau_{i}=\tau_{f}, \quad i=2,3 \ldots N_{3}$,

and

$\tau_{i}=\frac{K_{\mathrm{II}}}{\sqrt{2 \pi x_{i}}}+\frac{2 G b}{(\kappa+1) \pi}\left[-\frac{1}{2 x_{i}}+\sum_{j=1, j \neq i}^{N_{\mathrm{T}}} \frac{\sqrt{x_{j}}}{\sqrt{x_{i}}\left(x_{i}-x_{j}\right)}\right]$,

where $x_{O b}$ and $x_{i}$ are the first and the $i$ th dislocation positions, respectively, $\tau_{i}$ is the shear stress acting on the $i$ th dislocation, including the $K_{\text {II }}$ field produced by the external loading, its own image stress and the stress of all other emitted dislocations both in region 1 and region 3 and their images. $G$ is the shear modulus, $b$ is the Burgers vector and $\tau_{f}$ is the lattice friction.

It should be noted that all emitted dislocations lie on the $x$-axis. The dislocation number is counted from right to left, the dislocation positions are $x_{i}\left(i=1,2, \ldots N_{3}\right)$ in region 3 and $x_{i}\left(i=N_{3}+1, \ldots, N_{\mathrm{T}}\right)$ in region 1 , respectively.

In the reference model, any movement of dislocations in region 1 should affect the distribution of the emitted dislocations in region 3 . The change of dislocation positions in region 3 conversely will offer the displacement increment to deformation of the reference model. If our simulations were 

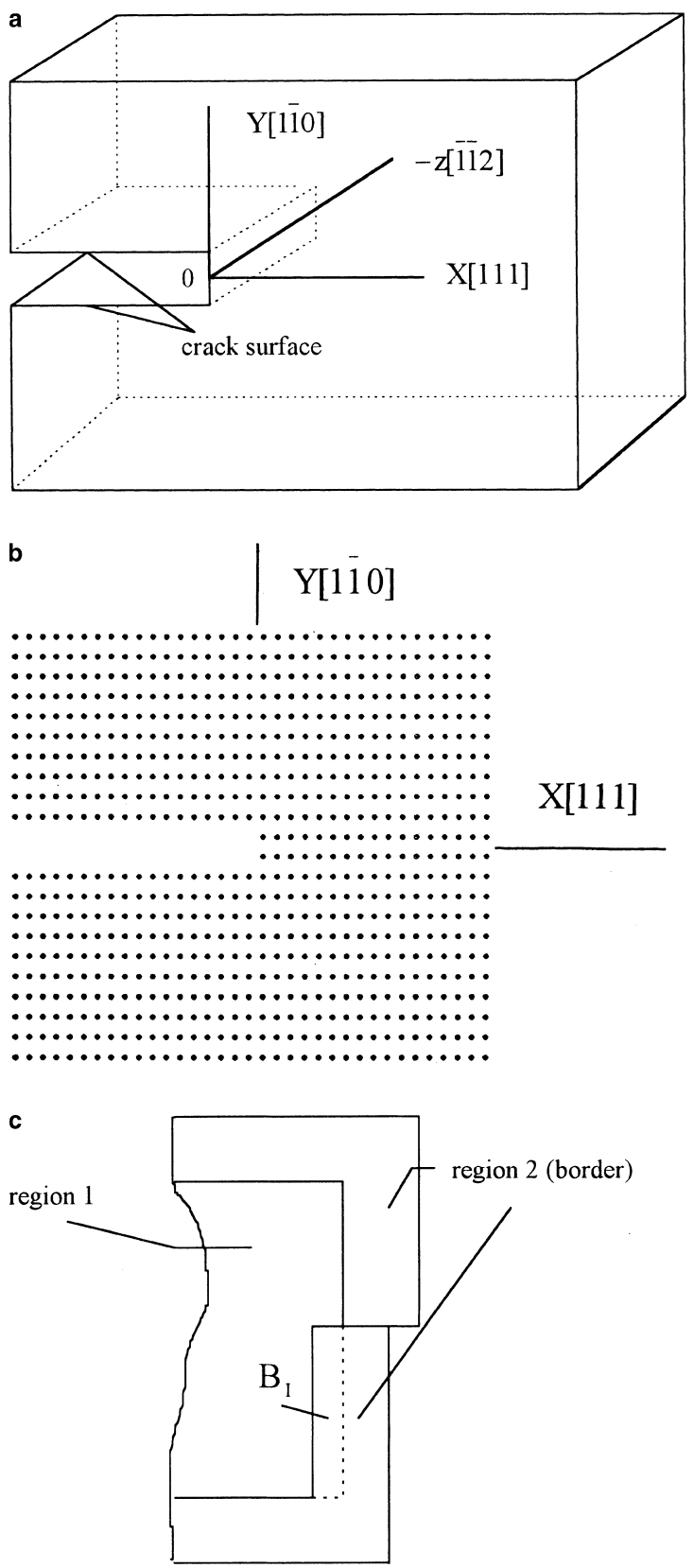

Fig. 2. (a) Schematic diagram for the simulated sample, (b) Atomic configuration near crack tip, (c) Schematic diagram of a dislocation penetrating through the border.

carried out in such a way, the non-linear Eqs. (2a) and ( $2 b)$ would have to be solved at each time step and the computational cost would be increased sharply. The emitted dislocations in region 3 pile up on the plastic zone which is far away from the crack tip, hence the effect of redistribution of the emitted dislocation in the plastic zone on the displacement of region 1 is quite small. In order to reduce the computation cost, a simple method is adopted in our simulation. As the nucleated dislocations move within region 1 , the positions of $N_{3}$ dislocations in region 3 are held fixed temporarily. But as the $\left(N_{3}+1\right)$ th dislocation penetrates through the boundary into region 3, Eqs. (2a) and (2b) are solved by the Newton-Raphson method, and the dislocation positions of region 3 will be redistributed. Consequently, the effect of the dislocation position redistribution in region 3 on the simulated sample is accounted in our stimulation.

\subsection{Basic formulas}

The displacement field $u_{l}^{A}$ of Eq. (1) is given by the linear elastic fracture theory,

$$
\begin{aligned}
& u_{x}^{A}=\frac{K_{\mathrm{II}}}{4 G} \sqrt{\frac{r}{2 \pi}}\left[(2 \kappa+3) \sin \frac{\theta}{2}+\sin \frac{\theta}{3}\right], \\
& u_{y}^{A}=\frac{K_{\mathrm{II}}}{4 G} \sqrt{\frac{r}{2 \pi}}\left[(2 \kappa+3) \cos \frac{\theta}{2}+\cos \frac{\theta}{3}\right],
\end{aligned}
$$

where $r$ is the distance from the crack tip, $\kappa=3-4 v$ and $v$ is the Poisson's ratio. The displacement field $u_{l}^{D}$ is given by the following equation

$2 G\left(u_{x}^{D}+i u_{y}^{D}\right)=\kappa \phi(z)-\omega(\bar{z})-(z-\bar{z}) \overline{\Phi(z)}$.

According to Lo [23], the complex functions $\Phi(z), \Omega(z), \phi(z)$ and $\omega(z)$ can be expressed as:

$\Phi(z)=\Omega(z)=\Phi_{0}(z)+\Phi_{R}(z)=\frac{B}{z-z_{0}} \sqrt{\frac{z_{0}}{z}}$,
$\phi(z)=\omega(z)=\int \Phi(z) \mathrm{d} x=B \ln \frac{\sqrt{z}-\sqrt{z_{0}}}{\sqrt{z}+\sqrt{z_{0}}}$,

where $B=(b G /(\kappa+1) \pi i)$. If the emitted dislocation lies on the $x$ axis, at $z_{0}=x_{0}$, the displacement field $u_{l}^{D}$ can be represented as

$$
\begin{aligned}
u_{x}^{D}+i u_{y}^{D}= & \frac{b}{2 i \pi(\kappa+1)}\left[\kappa \ln \frac{\sqrt{z}-\sqrt{x_{0}}}{\sqrt{z}+\sqrt{x_{0}}}\right. \\
& \left.-\ln \frac{\sqrt{\bar{z}}-\sqrt{x_{0}}}{\sqrt{\bar{z}}+\sqrt{x_{0}}}-(z-\bar{z}) \frac{1}{z-x_{0}} \sqrt{\frac{x_{0}}{z}}\right] .
\end{aligned}
$$


Compared to the existing models of atomistic core/ continuum $[18,20,22]$, our model is quite simple and easy to implement.

\section{Calculation method}

1. Interatomic potential: An 'N-body' potential has been proposed by Tang et al. [24] in which the embedding energy function was obtained by means of a numerical experiment for uniaxial tension. The total energy $E_{\mathrm{T}}$ of an assembly of atoms at position $r_{i}$ is given by

$E_{\mathrm{T}}=\frac{1}{2} \sum_{i, j, i \neq j} \phi\left(r_{i j}\right)+\sum_{i} F\left(\frac{\rho_{i}}{\rho_{e}}\right)$

where $r_{i j}$ is the distance between atoms $i$ and $j$, $\phi\left(r_{i j}\right)$ is the pair-potential, $F\left(\rho_{i} / \rho_{e}\right)$ is the embedding energy function. For a Mo crystal, the functions $\phi\left(r_{i j}\right)$ and $F^{\prime}\left(\rho_{i} / \rho_{e}\right)$ can be expressed by following equations [19]:

$$
\begin{aligned}
\phi\left(\frac{r_{i j}}{r_{1 e}}\right)= & B_{0}+B_{1}\left(\frac{r_{i j}}{r_{1 e}}-1\right) \\
& +B_{2}\left(\frac{r_{i j}}{r_{1 e}}-1\right)^{2}+B_{3}\left(\frac{r_{i j}}{r_{1 e}}-1\right)^{3} \\
& +H\left(r_{i j}-r_{2 e}\right)\left[B_{4}\left(\frac{r_{i j}}{r_{l e}}-1\right)^{3}\right. \\
& \left.+B_{5}\left(\frac{r_{i j}}{r_{1 e}}-1\right)^{3}\left(\frac{r_{c}}{r_{2 e}}-1\right)\right] /\left(\frac{r_{c}}{r_{2 e}}-1\right)^{3},
\end{aligned}
$$

and

$$
F^{\prime}\left(\rho / \rho_{e}\right)=\left\{\begin{array}{c}
-10 \sqrt{0.0515-\left(\rho / \rho_{e}-0.778\right)^{2}}+1.9, \\
0.68 \leqslant \rho / \rho_{e} \leqslant 0.775 \\
-0.72\left(\rho / \rho_{e}-0.775\right)-0.3 \\
0.775 \leqslant \rho / \rho_{e} \leqslant 0.83 \\
-10 \sqrt{0.397-\left(\rho / \rho_{e}-0.88\right)^{2}}+0.59, \\
0.83 \leqslant \rho / \rho_{e} \leqslant 1.01,
\end{array}\right.
$$

where $\rho_{i}$ is the atomic electron density, $r_{1 e}$ and $r_{2 e}$ are the first- and second-neighbor atom distance, respectively. The coefficients $B_{i,}(i=1, \ldots 6)$ in the Eq. (8) were given in Ref. [19]. $H\left(r_{i j}-r_{2 e}\right)$ is the step function and $\rho_{e}$ is the atom electron density of equilibrium.

2. Atomic lattice geometry: A parallelepiped with a slit is used as the simulated sample in the present calculation. The coordinate system is selected to be $x, y$ and $z$ axes along the [ [ $\left.\begin{array}{lll}1 & 1 & 1\end{array}\right],\left[\begin{array}{lll}1 & 1 & 0\end{array}\right]$, $\left[\begin{array}{lll}1 & 1 & \overline{2}\end{array}\right]$, respectively. The periodicity along [llll 1101$]$ is $\sqrt{3} a_{0} / 2$ in three layers, the length is $500 \times(\sqrt{3} / 6) a_{0} ;$ the periodicity along $\left[\begin{array}{lll}1 & \overline{1} & 0\end{array}\right]$ is $\sqrt{2} a_{0}$ in two layers and the length is $40 \times(\sqrt{2} / 2) a_{0}$; the periodicity along $\left[\begin{array}{lll}1 & 1 & \overline{2}\end{array}\right]$ is $\sqrt{6} a_{0}$ in six layers and the length is $6 \times(\sqrt{6} / 6) a_{0}$; the left side of the boundary to the crack tip is $120 \times(\sqrt{3} / 6) a_{0}$; the separation of the upper and lower crack planes is taken to be $\sqrt{2} a_{0}$; the number of atoms in the present simulation is about 20000 , see Fig. 2(a) and (b). The slip plane is coplanar with the crack, so emerging dislocations are of edge character relative to the tip under the pure shear loading.

3. The boundary condition: The flexible boundary condition described in Section 2 is adopted here.

4. The calculation method: The leapfrog algorithm [25] is used in this paper, the loading rate is $\dot{K}_{\text {II }}=0.0706\left(\mathrm{M} \mathrm{Pam}^{1 / 2} / \mathrm{ps}\right)$, the stress intensity factor $K_{\mathrm{II}}$ is chosen as the loading parameter. The time step is $1.256 \times 10^{-14} \mathrm{~s}$.

5. The temperature condition: The initial velocities of the atoms follow the Maxwellian distribution corresponding to a given temperature. We suppose that the simulated system is connected to a big heat source with a certain temperature which provides enough heat energy to maintain the system at a fixed value throughout the duration of the runs. This means that any little perturbation due to loading continuously or the dislocation emission can be neglected $[25,26]$, here $T=50 \mathrm{~K}$.

\section{Results and discussion}

The positions of eight fully emitted dislocations versus time are plotted in Fig. 3. The MD simulated results show that a full dislocation is dissociated into three partial dislocations in a bcc crystal of Mo (see Fig. 3 and Fig. 6), but into two partial dislocations in fcc crystal $(\mathrm{Cu})$ [10]. 


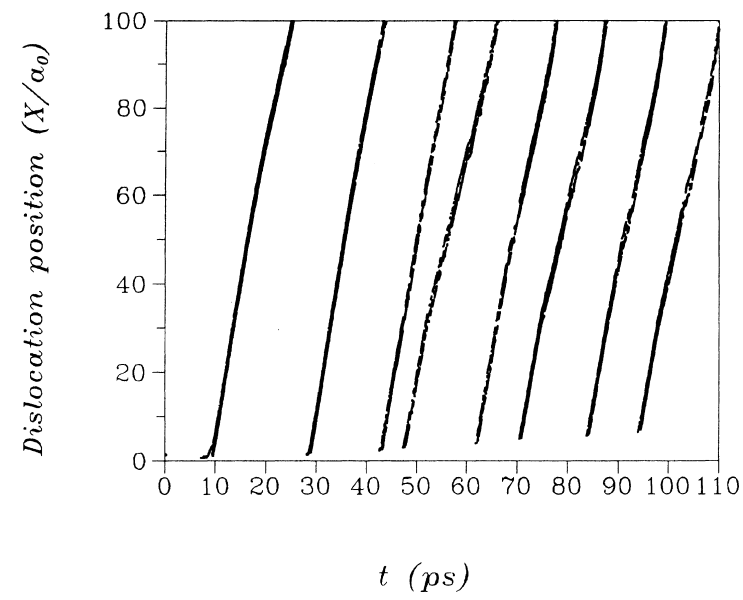

Fig. 3. Distance of the dislocation position from the crack tip plotted versus time.

Additionally the distance among the three partial dislocations in the bcc crystal (Mo) is shorter than that in an fcc crystal; they are about 4 and $20 a_{0}$, respectively. The eight full dislocations ( 24 partial dislocations) slip into region 3 smoothly and are assumed to pile up at a distance of $5000 a_{0}$ from the crack tip.

When the first three partial dislocations have been emitted, we pause to load temporarily, and keep the load unchanged in the reference model. The nucleated dislocation is subjected to the repulsive force from the $K_{\mathrm{II}}$ external stress field and moves away along the slip plane with high velocity. Within a distance of $5 a_{0}$ from crack tip, the dislocation is in an accelerative state and reaches a constant velocity quickly. Within a range of between 5 and $100 a_{0}$, all the eight full dislocations move at almost the same velocity, about $1800-1950 \mathrm{~m} / \mathrm{s}$, which is less than the shear wave velocity of the metal crystal Mo that is about $3500 \mathrm{~m} / \mathrm{s}$.

On a time scale of picoseconds, the time interval of emitting dislocations is not homogeneous, the longest time interval is $20 \mathrm{ps}$ between the first full dislocation and the second full dislocation, the shortest is 6 ps between the third and the fourth full dislocation. But on a time scale of $\mu$ s or ms, the dislocation emission is continuous. The simulated result is in good agreement with experimental observations [27].
As the emitted dislocations are far away from crack tip, the interaction between the dislocation and the crack becomes less violent, but the interaction of two dislocations is the same as before, so the latter interaction appears more important. The result shows that the spatial interval between the third and fourth full dislocations is from narrow to wide, and becomes more homogeneous gradually.

The external stress intensity factor $K_{\mathrm{II}}$ versus time, $t$, is shown in Fig. 4. Comparing Fig. 4 with Fig. 3, we observe that at $t=29 \mathrm{ps}$, the first three partial dislocations slip into region 3 . Because no dislocation is found in the region 1, so we should load again at the same loading rate as before. As $K_{\text {II }}$ reaches $0.801 \mathrm{M} \mathrm{Pam}^{1 / 2}$, the second full dislocation has been emitted. Compared with the critical stress intensity factor $K_{\mathrm{II}_{e}}\left(K_{\mathrm{II}_{e}}=0.668 \mathrm{M} \mathrm{P}\right.$ $\mathrm{am}^{1 / 2}$ ) the loading increment is about $10 \%$ of $K_{\mathrm{II}_{e}}$. From the third full dislocation to the eight full dislocation the loading increment is less than $1 \%$ of $K_{\mathrm{II}_{e}}$. The curve of the dislocation distance from the crack tip versus the external stress intensity factor $K_{\mathrm{II}_{e}}$ is depicted in Fig. 5.

The atomic configuration near the crack tip is showed in Fig. 6, three partial edge dislocations can be observed clearly in the slip plane. At this time, $K_{\mathrm{II}}=0.802 \mathrm{M} \mathrm{Pam}^{1 / 2}$, corresponding to the fourth full dislocation. Although a pure mode II loading is applied, the moving-away direction of the partial dislocation is not along the $x$-direction [ $\left.\begin{array}{lll}1 & 1 & 1\end{array}\right]$, but along a certain angle from $x$-direction

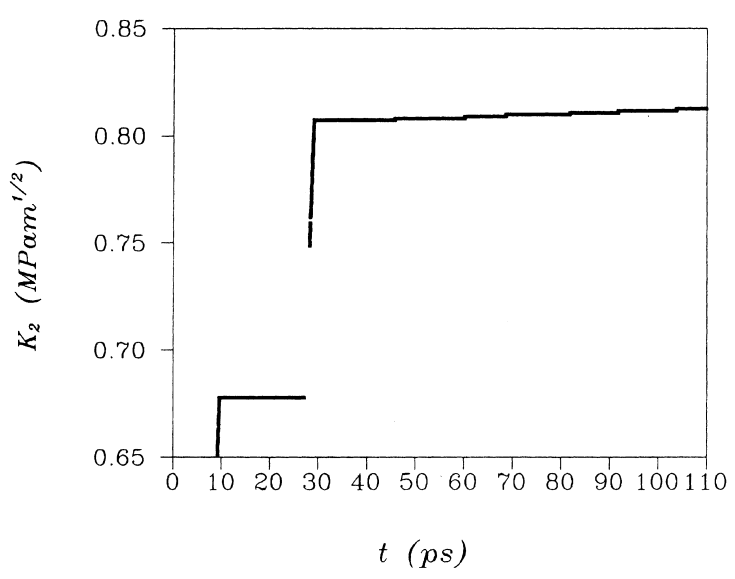

Fig. 4. Stress intensity factor versus time. 


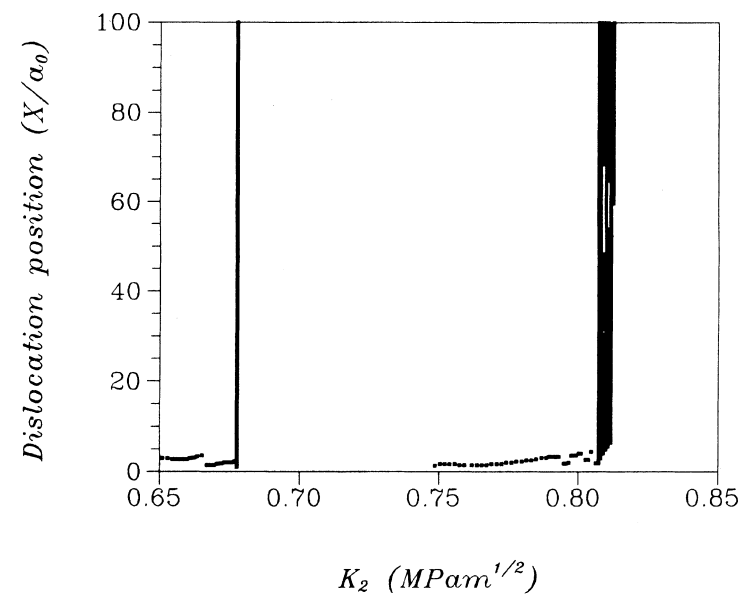

Fig. 5. Distance of the dislocation position from the crack tip plotted against the stress intensity factor.

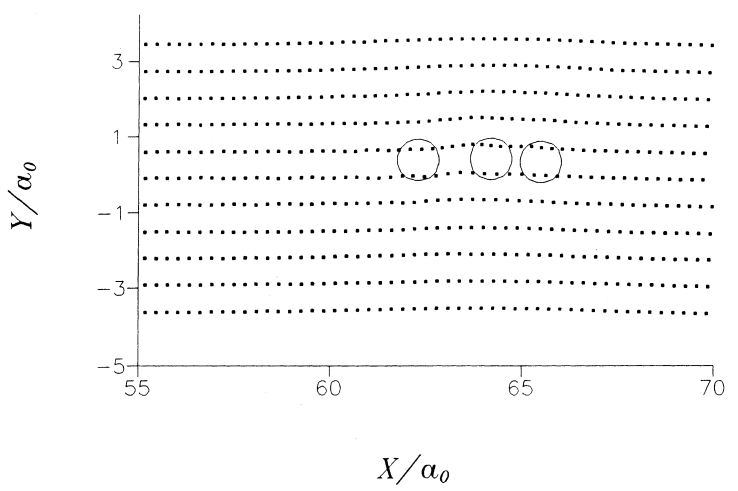

Fig. 6. Atomic configuration, plotted for the position of dislocation, $K_{I I}=0.802 \mathrm{M} \mathrm{Pam}^{1 / 2}$.

on $x-y$ plane $\left(\begin{array}{lll}1 & 1 & 0\end{array}\right)$ where the resistance force encountered by partial dislocation is very small. The determination of the exact value of the angle is difficult because the simulated atoms are in a dynamic state, but the moving away direction of the full dislocation is in the $x$-direction.

The shear stress along the propagating crack is showed in Fig. 7 at a load level of $K_{\text {II }}=0.802 \mathrm{M} \mathrm{P}$ $\mathrm{am}^{1 / 2}$. The partial dislocations of the fourth full dislocation are between 60 and $65 a_{0}$. It can be seen that our result is in good agreement with the elasticity solution. The stress on atomic scale can be evaluated through an average of the force acting on each atom from the surrounding [28],

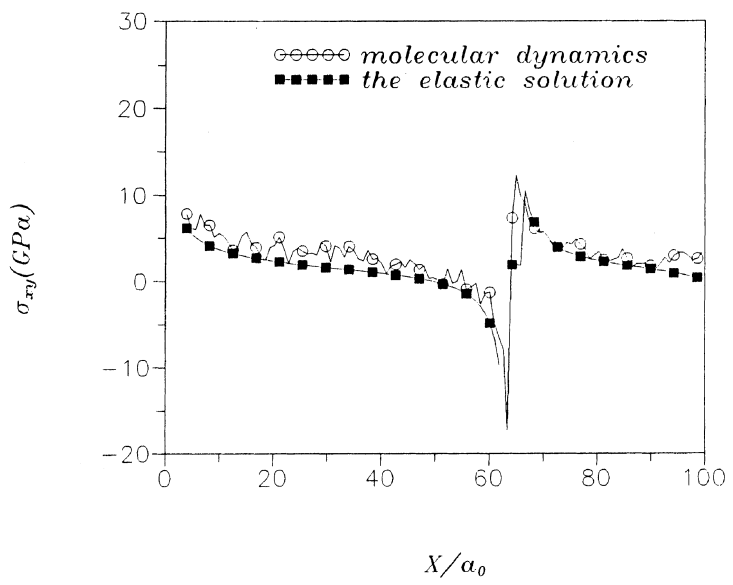

Fig. 7. After a dislocation is emitted from the crack tip, the result of the MD simulation is compared to the elastic solution, the shear stress plotted against distance from crack tip.

$\sigma_{k l}=\frac{1}{\Omega_{0}} \sum_{i, j, i \neq j}\left[\frac{1}{2} \phi^{\prime}\left(\frac{r_{i j}}{r_{1 e}}\right)+F^{\prime}\left(\frac{\rho_{i}}{\rho_{e}}\right) \frac{\rho_{i, j}^{\prime}}{\rho_{e}}\right] \frac{r_{i j}^{k} r_{i j}^{l}}{r_{i j}}$,

where $\Omega_{0}$ is atomic volume, $k$ and $l$ are the coordinate components. The linear elastic solution of the interaction field produced by the singularity of the crack tip and the edge dislocation with Burger's vector $b$ is given by

$\sigma_{x y}=\frac{K_{\mathrm{II}}}{\sqrt{2 \pi x}}+\sum_{i} \frac{G}{2 \pi(1-v)} \sqrt{\frac{x_{i}}{x}} \frac{b}{x-x_{i}}$.

The stress produced by Eq. (11) at the dislocation core has a singularity, a modification according to the Peierls formula is introduced around the dislocation center [29]

$\sigma_{x y}=\frac{G b}{2 \pi(1-v)} \frac{x}{x^{2}+\xi^{2}}$,

where $\xi$ the radius of dislocation core.

According to Rice [1], after the dislocation emitted from the crack tip, the interaction of the dislocation and the crack leads to a decrease of the local stress intensity factor $K_{\mathrm{II}}^{\mathrm{tip}}$ that is given by

$K_{\mathrm{II}}^{\mathrm{tip}}=K_{\mathrm{II}}-2 \sqrt{2 \pi} A b \sum_{i=1}^{n} \frac{1}{\sqrt{x_{i}}}$ 
The result in Fig. 8 shows that as $K_{\mathrm{II}}=0.801 \mathrm{M} \mathrm{P}$ $\mathrm{am}^{1 / 2}$, the second full dislocation is emitted. It means that from the first full dislocation to the second full dislocation, the incremental amount of external loading is up to approximately $10 \%$ of $K_{\mathrm{II}_{e}}$, but in the following dislocation emission, the incremental amount of external loading is quite smaller. There may be two factors corresponding to the difference of loading amount between the first and second full dislocation emission. One is that as the external load is at $K_{\mathrm{II}}=0.668 \mathrm{M} \mathrm{Pam}^{1 / 2}$, the deformation of the crack tip region is still in a sable state after the first full dislocation piles up at a distance of $5000 a_{0}$ from the crack tip. No evidence of nucleation of the next full dislocation near the crack tip is observed within the following relaxation of 100 time step, unless reload is carried out again at infinity in the reference model. But as the external load reaches $K_{\mathrm{II}}=0.801 \mathrm{M} \mathrm{P}$ am $^{1 / 2}$, the deformation of the discrete atomic region is in the unstable state in which more than two full dislocations can be emitted continuously. The other factor is that after the first full dislocation slips into region 3 the reloading rate is too high leading to such a difference of the loading amounts.

Based on Eq. (13), the local intensity factor $K_{\mathrm{II}}^{\mathrm{tip}}$ can be obtained, decreasing gradually as shown in Fig. 8. This means that in the study of the

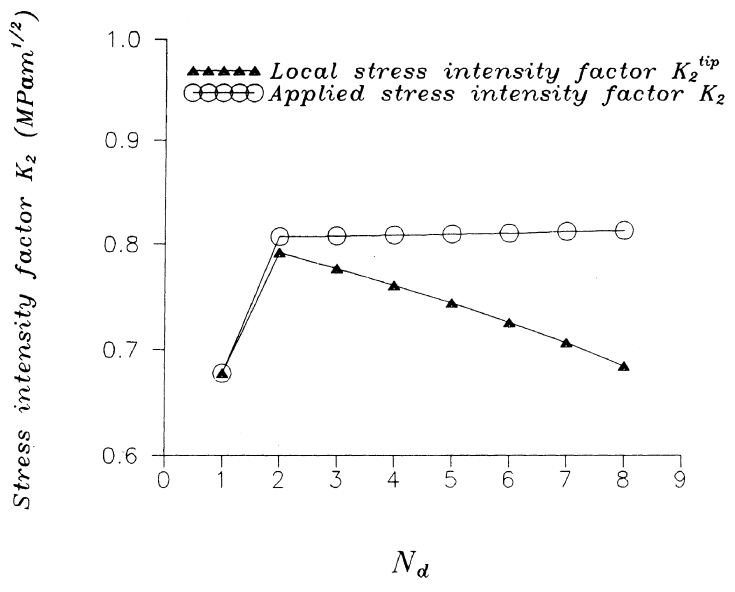

Fig. 8. External stress intensity factor compared to the local stress intensity factor, the stress intensity factor versus dislocation number is plotted. dislocations emission from the crack tip, the local stress intensity factor cannot simply be used as a control parameter.

Eqs. (2a) and (2b) can be solved with the Newton-Raphson method with $\left(\tau_{f} / G\right)=0.00227$. The positions of the emitted dislocations in region 3 are shown in Fig. 9. As the total number of the emitted dislocations increase, the positions of dislocations pileup in region 3 will be changed. The effect of redistribution of the dislocation position on the MD simulated sample is reflected in our simulation.

The atomic configuration near the right boundary is plotted in Fig. 10. The left trace of 24 partial dislocations slipping into the region $3 \mathrm{can}$ be observed clearly. From Fig. 10, we can image that the slipping dislocations may pileup in front of an obstacle where there is a grain boundary or other imperfections, slip out of the crystal, stay at the crystal surface, so that some morphology, such as steps or ledges can be observed on the crack surface or the crystal surface.

\section{Conclusion}

(1) Both a correlative reference model and a flexible displacement boundary have been presented which offer several advantages over

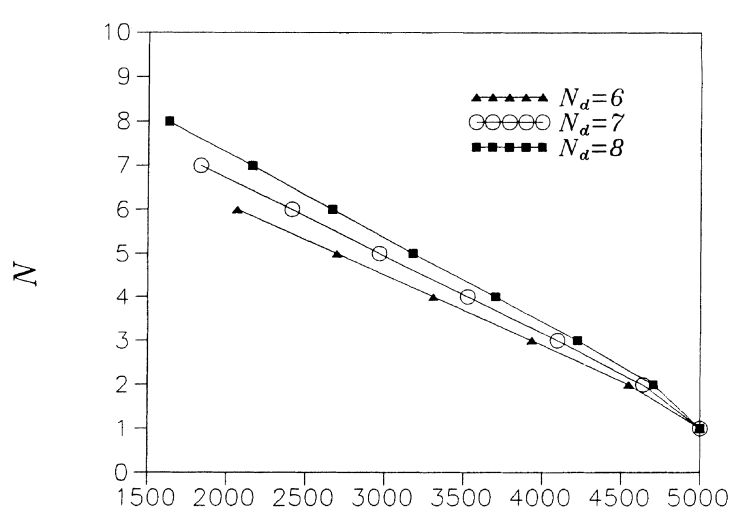

$$
X / \alpha_{0}
$$

Fig. 9. Dislocation number versus dislocation position, $\left(\tau_{f} / G\right)=0.00227$. 


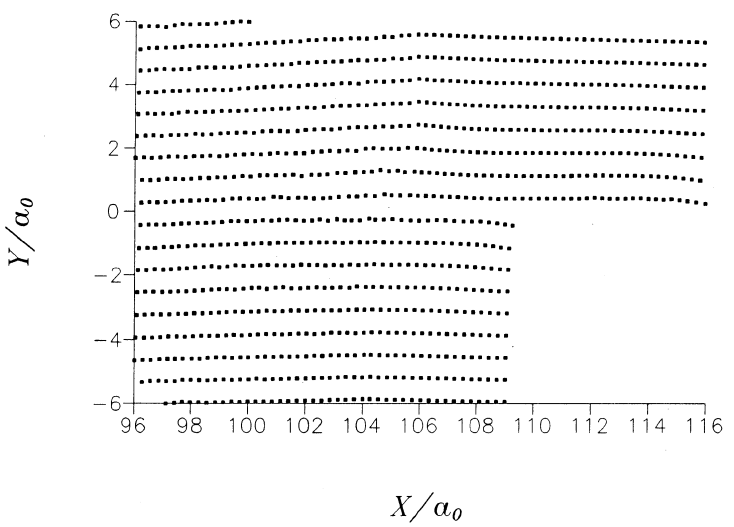

Fig. 10. Atomic configuration of the right boundary of the simulated sample.

previous schemes. Especially, the problem of a dislocation penetrating through the border has been solved successfully. Although the size of the simulated atomistic core is finite, the number of dislocation emission can increase without restriction.

(2) A molecular dynamics simulation was carried out for a Mo crystal. After the second dislocation is emitted, the dislocation can be emitted from the crack tip continuously, as the applied load increases a little.

(3) Within a distance of $5 a_{0}$ from the crack tip, the dislocation is in an accelerative state, and reaches a stable velocity quickly; within the range of between 5 and $100 a_{0}$, the dislocation velocity is nearly constant, about $1800-1950 \mathrm{~m} / \mathrm{s}$, which is in agreement with that predicated in Ref. [30].

\section{Acknowledgements}

The research presented here was supported by the National Natural Science Foundation of China (No. 19392300-1).

\section{References}

[1] J.R. Rice, R. Thomson, Philo. Mag. 29 (1974) 73.

[2] J.E. Sinclair, M.W. Finnis, in: R.M. Latanision, J.R. Pickens (Eds.), Atomics of Fracture, Plenum Press, New York, 1983.

[3] I.H. Lin, J.P. Hirth, Philos. Mag. A 50 (1984) L43.

[4] J.R. Rice, J. Mech. Phys. Solids 40 (1992) 239.

[5] T.C. Wang, Int. J. Fract. 69 (1995) 295.

[6] H.C. Choi, A.F. Schwartzman, K.S. Kim, Mat. Res. Symp. Proc. 239 (1992) 419.

[7] R. Bullough, Bull. Am. Phys. Soc. A 10 (1965) 323.

[8] R.M.J. Cotterill, M. Doyama, Phys. Rev. 145 (1966) 465.

[9] M.I. Baskes, M.S. Daw, S.M. Foiles, Mater. Res. Soc. Symp. Proc. 141 (1989) 31.

[10] Y.W. Zhang, T.C. Wang, Q.H. Tang, Acta Mechanica Sinica 10 (1995) 150.

[11] Y.W. Zhang, T.C. Wang, Q.H. Tang, J. Appl. Phys. 77 (1995) 2393.

[12] K.S. Cheung, S. Argon, S. Yip, J. Appl. Phys. 69 (1991) 2088.

[13] P.C. Gehlen, M.F. Kanninen, in: M.F. Kanninen, W.F. Alder, A.R. Rosenfield, R.I. Jaffee (Eds.), Inelastic Behavior of Solids, McGraw-Hill, New York, 1970, p. 587.

[14] M.F. Kanninen, P.C. Gehlen, Int. J. Fract. Mech. 1 (1971) 471.

[15] J.E. Sinclair, J. Appl. Phys. 42 (1971) 5321.

[16] P.C. Gehlen, J.P. Hirth, R.G. Hoagland, M.F. Kanninen, J. Appl. Phys. 43 (1972) 3921.

[17] H. Kitagawa, A. Nakatani, Proceeding of JSME Ser. A 59-557 (1993) 256.

[18] M. Mullins, M.A. Dokainish, Philo. Mag. A 46 (5) (1982) 771.

[19] M. Mullins, Int. J. Fract. 24 (1984) 189.

[20] S. Kohlhoff, P. Gumbsch, H.F. Fischmeister, Philo. Mag. A 64 (4) (1991) 851.

[21] P. Gumbsch, J. Mater. Res. 10 (11) (1995) 2897.

[22] H. Tan, W. Yang, Acta Mech. Sinica 10 (2) (1994) 150.

[23] K.K. Lo, J. Appl. Mech. 45 (1978) 797.

[24] Q.H. Tang, T.C. Wang, Y.W. Zhang, Acta Mech. Sinica 12 (4) (1996) 358.

[25] D.W. Heermann, Computer Simulation Methods in Theoretic Physics, 2nd ed., Springer, Berlin, 1990.

[26] S. Nose, in: M. Meyer, V. Pontikis (Eds.), Computer Simulation in Materials Science, vol. 21, 1991.

[27] J.A. Horton, S.M. Ohr, J. Mater. Sci. 17 (1982) 3140.

[28] I. Alber, J. L. Bassani et al., Phil. Trans. R. Soc. Lond. A 339 (1992) 555.

[29] R.E. Peierls, Proc. Phys. Soc. 52 (1940) 34.

[30] J.J. Gilman, J. Appl. Phys. 31 (1960) 2208. 